\title{
Características e potencialidades dos frutos do Cerrado na indústria de alimentos
}

\section{Characteristics and potentialities of Savanna fruits in the food industry}

\author{
Amanda Figueiredo Reis $^{1}$ (D), Marcio Schmiele ${ }^{1 *}$ [D \\ ${ }^{1}$ Universidade Federal dos Vales do Jequitinhonha e Mucuri (UFVJM), Instituto de Ciência e Tecnologia (ICT), \\ Diamantina/MG - Brasi
}

*Corresponding Author: Marcio Schmiele, Universidade Federal dos Vales do Jequitinhonha e Mucuri (UFVJM), Instituto de Ciência e Tecnologia (ICT), Rodovia MGT-367, km 583, 5000, Alto do Jacuba, ICT sala 349,

CEP: 39100-000, Diamantina/MG - Brasil, e-mail: marcio.sc@ict.ufvjm.edu.br

Cite as: Reis, A. F., \& Schmiele, M. (2019). Characteristics and potentialities of Savanna fruits in the food industry

Brazilian Journal of Food Technology, 22, e2017150. https://doi.org/10.1590/1981-6723.15017

\section{Resumo}

O Cerrado é o segundo maior bioma brasileiro e apresenta grande diversidade de frutos que possuem alto valor nutricional, sabor e aroma característicos, compostos bioativos com propriedades antioxidantes e apelo saudável. O mercado consumidor visa a produtos com apelo natural e funcional, e, pelo fato de haver grandes perdas póscolheita dos frutos do cerrado, cabe à indústria de alimentos aliar tais propriedades dos frutos à elaboração de novos produtos com valor agregado e maior tempo de vida de prateleira. O presente trabalho apresenta uma revisão com o objetivo de listar e caracterizar os frutos do cerrado (pequi, bocaiuva, mangaba, cagaita, baru, murici, mama-cadela, buriti, araticum e guabiroba), apresentando estudos com possíveis aplicações na indústria de alimentos.

Palavras-chave: Compostos bioativos; Coprodutos; Bioma; Pequi; Araticum; Cagaita; Baru.

\begin{abstract}
The Savanna is the second biggest biome in Brazil and shows a great diversity of fruits with high nutritional value, unique taste and flavour and bioactive compounds with antioxidant properties and health benefits. The consumer market targets products with natural and functional claims and due to the great post-harvest losses of Savanna fruits, the food industry should ally the properties of these fruits and manufacture new products with aggregated values and extended shelf lives. This paper presents a literature review aiming to list and characterize the Savanna fruits (pequi, bocaiuva, mangaba, cagaita, baru, murici, mama-cadela, buriti, araticum and guabiroba) including some studies with possible applications in food industries.
\end{abstract}

Keywords: Bioactive compounds; Coproducts; Biome; Pequi; Annona; Cagaita; Baru.

\section{Introdução}

Conhecida como um dos biomas com maior biodiversidade do mundo, o Cerrado Brasileiro possui formação savânica e corresponde a uma área aproximada de 2,0 milhões de $\mathrm{km}^{2}$, representando em torno de $23 \%$ do território nacional. Esta área abrange o sul do Mato Grosso, o norte do Piaú, o oeste da Bahia, o sul 
do Maranhão, os Estados de Goiás, Tocantins, Mato Grosso do Sul, Minas Gerais, Rondônia e São Paulo, e o Distrito Federal (Soares et al., 2017).

A maior parte deste bioma possui condições edafoclimáticas particulares. O clima dominante é o tropical sazonal, apresentando precipitação pluviométrica anual média de $1.500 \mathrm{~mm}$, sendo que cerca de $90 \%$ das chuvas ocorrem entre os meses de outubro e março, definindo dois períodos - o chuvoso e o de estiagem. A temperatura varia entre $22{ }^{\circ} \mathrm{C}$ e $27{ }^{\circ} \mathrm{C}$, e a umidade relativa do ar alcança taxas entre 38 e $40 \%$ no inverno seco, enquanto no período chuvoso, a umidade é elevada, atingindo $97 \%$. A vegetação do bioma é caracterizada como aberta ou floresta semidecidual, possui habitats xerofíticos, mésicos e enclaves com regiões úmidas, caracterizada por árvores de médio porte, galhos e troncos retorcidos, raízes densas e acomodadas a uma menor pluviosidade (Conceição, 2012; Mendes et al., 2012; Almeida Júnior et al., 2014).

O Cerrado apresenta fauna e flora extremamente rica, ressaltando as classes de frutos presentes, com características sensoriais intrínsecas e com alta qualidade nutricional, o que os tornam atraentes para serem explorados, pesquisados e comercializados (Angella, 2014; Morzelle et al., 2015). Além disso, com os avanços das tecnologias de produção, o uso da irrigação, o melhoramento genético e a necessidade de um melhor aproveitamento dos frutos para diminuir os desperdícios, o cultivo destes frutos pode vir a ser explorado fora do bioma original. Como exemplo, temos a produção de uva, pera e maçã sendo realizada na Região Nordeste do país, frutos que eram produzidos somente na região fria do País.

As plantas do Cerrado são adaptadas às condições ambientais distintas: extensos períodos de seca, outrora períodos de alta precipitação, solos pobres, grande ocorrência de incêndios e alta incidência de radiação UV. Diante disto, há a necessidade de as plantas utilizarem mecanismos de defesa para se protegerem de agentes físicos, químicos e biológicos, no decorrer do seu processo evolutivo. Com isso, pode-se associar a presença de compostos bioativos nas mesmas.

A caracterização dos compostos bioativos em frutos do Cerrado é de grande relevância para a busca de fontes alternativas e que possam agrupar atributos desejáveis (propriedades antioxidantes, antimicrobianas, anticarcinogênicas, antidegenerativas e retardadoras de envelhecimento). Este incremento pode ocorrer na formulação de novos produtos, ou mesmo na ingestão in natura, uma vez que tais compostos são de interesse tanto para a indústria de alimentos quanto para a de fármacos e de cosméticos (Luzia, 2012).

Nas etapas de colheita, transporte, armazenamento e comercialização, há muitas perdas e geração de coprodutos (Weiss \& Santos, 2012). A FAO (Food and Agriculture Organization of the United Nations, 2012) aponta que, em breve, será imprescindível o aumento da produção de alimentos em $60 \%$ até 2050 , devido às mudanças climáticas e ao constante crescimento da população. A busca por soluções para minimizar as perdas e agregar valor aos coprodutos é essencial. Para isso, são necessários estudos científicos e tecnológicos, que fundamentem o uso de forma competente, economicamente viável, segura e sem impactos negativos.

Segundo dados do Brazil Food Trends 2020 (Instituto de Tecnologia de Alimentos, 2010), dentre as tendências de mercado, destaca-se a preferência por produtos com qualidades sensoriais e nutricionais que proporcionem saudabilidade e bem-estar, cabendo às indústrias de alimentos se adaptar a esses segmentos de mercado, buscando novas formulações e produtos alimentícios inovadores (Oliveira Júnior et al., 2016). Desta forma, objetivamos explorar as principais características dos principais frutos do Cerrado e o potencial uso no processamento de alimentos.

\section{Frutos do cerrado}

Dez frutos do cerrado com expressiva produção foram selecionados, sendo: pequi, bocaiuva, mangaba, cagaita, baru, murici, mama-cadela, buriti, araticum e guabiroba. O levantamento de dados e informações foi realizado em bases de dados científicos (Science Direct, Web of Science, Scopus, Scielo - Scientific Electronic Library Online e no portal de periódicos da Capes) e no catálogo de Teses e Dissertações da Capes. 
A seleção dos trabalhos de maior relevância foi feita através do refino das informações, tomando-se por critérios as publicações nos últimos sete anos, e os frutos que foram selecionados a partir do maior número de trabalhos encontrados.

\subsection{Pequi}

O pequi (Caryocar Brasiliense Camb.) é um fruto do tipo drupa com epicarpo espesso de coloração esverdeada a tons de roxo, mesocarpo externo de cor esbranquiçada e mesocarpo interno (polpa) de cor amarela, coberta por um endocarpo espinhoso, o qual possui a função de proteger a amêndoa (uma a quatro, encontradas na parte central do fruto) (Gonçalves et al., 2015). A amêndoa é revestida por um tegumento de coloração marrom (Lima et al., 2007). A frutificação do pequi ocorre entre os meses de janeiro e março, sendo encontrado em toda a extensão do Cerrado Brasileiro, figurando o Estado de Minas Gerais o principal produtor (73\% da produção) (Leão et al., 2017).

A polpa é rica em lipídeos (principalmente ácidos graxos oleico e palmítico) e fibras alimentares, como pectina (ramnogalacturanas) e hemicelulose (arabnogalactanas, xilanas e glucomanas). A coloração amarela é devida à presença de carotenoides ( $\alpha$ e $\beta$-caroteno) com teores em torno de 2 a $3 \mathrm{~g} / 100 \mathrm{~g}$ de polpa. Possui ainda compostos fenólicos (polifenóis, proantocianidinas não extraíveis), componentes bioativos com expressiva capacidade antioxidante (Lima et al., 2007; Leão et al., 2017), tanto para sequestro de radicais livres como para oxirredução, e vitamina $\mathrm{C}(100 \mathrm{mg} / 100 \mathrm{~g}$ de polpa).

O óleo da amêndoa apresenta elevado teor de ácidos graxos poli-insaturados e ácidos graxos essenciais ( $\omega-3$ e $\omega-6)$, além de compostos bioativos com efeito benéfico sobre indução do processo oxidativo desencadeado por radicais livres, proteção hepatotóxica e ação anti-inflamatória, conforme relatado por Torres et al. (2016).

\subsection{Buriti}

O buriti (Mauritia flexuosa L.f.) é um fruto com forma elipsoidal, coloração castanho-avermelhado, podendo ser isento ou apresentar até dois caroços. A camada externa é revestida por escamas brilhantes e, abaixo destas, há uma pasta amarela, que recobre o caroço - comumente denominada de bucha. $\mathrm{O}$ comprimento de cada fruta varia entre 4 e $5 \mathrm{~cm}$, com massa de aproximadamente 20 a $40 \mathrm{~g}$ (Sampaio, 2011; Sales, 2016). A frutificação do buriti ocorre entre os meses de dezembro e junho, na maioria do território coberto pelo Cerrado (Carneiro \& Carneiro, 2011).

A polpa do fruto é comestível e de coloração alaranjada devida ao teor de carotenoides presentes, principalmente de $\beta$-caroteno. O buriti é rico em vitaminas $\mathrm{B}, \mathrm{C}$ e E, possui alto teor de fibras alimentares, polifenóis, lipídeos com ácidos graxos insaturados e ferro (Pessôa, 2017). O óleo presente na polpa do buriti apresenta alta quantidade de ácidos graxos monoinsaturados, cerca de $73 \%$, principalmente de ácido oleico (72\%) (Aquino et al., 2012b). Além disso, apresenta teor considerável de ácidos graxos saturados, aproximadamente $25 \%$, sobretudo de ácido palmítico (22\%) (Cruz et al., 2016).

\subsection{Mangaba}

A mangaba (Hancornia speciosa Gomes) é um fruto do tipo baga, geralmente possuindo de duas a 15 sementes, podendo até mesmo apresentar 30 sementes discoides, achatadas e com coloração castanhoclaro, com diâmetros de 7 a $8 \mathrm{~mm}$. O formato varia em elipsoidais ou arredondados, possuindo diâmetro diversificado entre 2,5 e $6,0 \mathrm{~cm}$. O exocarpo apresenta tons amarelados ou esverdeados, com pigmentação vermelha ou sem pigmentação. A polpa, carnoso-viscosa, é amarela, ácida e adocicada (Maia, 2016). A frutificação da mangaba ocorre entre os meses de outubro e dezembro, sendo vastamente encontrada nas áreas de tabuleiros e baixadas litorâneas da Região Nordeste (Nascimento et al., 2014). Trata-se de um fruto de alto valor nutricional, com quantidades significativas de pró-vitamina A e as vitaminas B1, B2 e C, e 
minerais, como ferro, fósforo e cálcio. O teor de proteínas da mangaba é superior ao encontrado na maioria das frutas comercializadas, sendo que, em 100 gramas de polpa, encontra-se, em média, 0,7 g de proteínas (Hansen, 2011).

\subsection{Cagaita}

A cagaita (Eugenia dysenterica) é um fruto de forma esférica. Quando maduro, apresenta coloração amarelo-claro e sabor ligeiramente ácido. O fruto apresenta formato oval, achatado ou elipsoide, com massa de 14 a 20 gramas, sendo o epicarpo membranoso e de brilho intenso, e o mesocarpo e o endocarpo, carnosos (Silva et al., 2015a, 2015b; Silva, 2016). A frutificação da cagaita ocorre aproximadamente um mês depois do florescimento (de agosto a setembro) (Almeida Júnior et al., 2014). É um fruto com teor significativo de fibras alimentares, vitaminas, minerais e baixo valor energético (cerca de 20 a $29 \mathrm{kcal}$ em 100 gramas). Possui alto teor de umidade, aproximadamente $95 \%$, e quantidades consideráveis de ácidos graxos essenciais, principalmente ácido linoleico ( $\omega-6)$, cerca de 10,5\%, e ácido linolênico ( $\omega$-3), cerca de 11,8\% (Silva, 2016).

\subsection{Araticum}

O fruto do araticum (Annona crassiflora Mart.) é do tipo baga, com formato circular achatado, de epicarpo rígido e, quando maduro, apresenta coloração marrom. Os frutos possuem diâmetros de aproximadamente $12 \mathrm{~cm}$ (Cardoso, 2011). A frutificação do araticum ocorre entre os meses de setembro e janeiro (Morais et al., 2017). A polpa é em forma de cone com coloração amarelo-claro, espessa, mole e reveste uma semente elíptica de coloração marrom-escuro (Cardoso, 2011). A polpa do fruto é fonte de ferro e de pró-vitamina A (Damiani et al., 2011). De acordo com Luzia (2012), a semente de araticum possui teor consideravelmente alto de óleo, o que admite extração em prensa contínua ou por batelada.

\subsection{Guabiroba}

O fruto da guabiroba (Campomanesia cambessedeana Berg.) é comestível, com sabor adocicado, polpa suculenta e do tipo baga, e possui formato globoso, apresentando diâmetros entre 15 e 20 mm. A coloração do fruto é amarelada ou alaranjada, no estádio maduro, com aroma cítrico agradável. $\mathrm{O}$ fruto pode apresentar de uma a seis sementes de coloração castanha, formato achatado e com três a oito milímetros de diâmetro (Albuquerque, 2016; Braga, 2017). A guabiroba se encontra em maior concentração no Estado de Goiás e a frutificação ocorre de setembro a dezembro, podendo prolongar-se até fevereiro (Alves et al., 2013).

A guabiroba possui alto teor de umidade e baixa quantidade de lipídeos, resultando em baixo valor energético, de aproximadamente $47 \mathrm{kcal} \mathrm{em} 100 \mathrm{~g}$ de polpa, além de possuir considerável teor de ácido cítrico e a presença de compostos fenólicos e $\beta$-caroteno, ambos com capacidade antioxidante (Alves, 2013).

\subsection{Murici}

O murici (Byrsonima crassifolia) é do tipo drupa, possui coloração amarelada, formato esférico e levemente achatado, apresenta diâmetro aproximado de 1,5 a 2,0 cm (Monteiro \& Pires, 2016). A floração do muricizeiro tem início no fim do mês de agosto e a frutificação, no final de setembro, podendo também ser em meados de janeiro a março, dependendo do índice pluviométrico no ano (Belisário \& Coneglian, 2013).

A casca possui sabor adstringente devido à presença de taninos e a polpa é carnosa e suculenta. O fruto é fonte de energia, lipídios, fibras alimentares, cálcio e vitamina C (84 mg/100 g), além de possuir componentes antioxidantes, como os compostos fenólicos e os carotenoides (Carvalho \& Nascimento, 2016). 


\subsection{Mama-cadela}

A mama-cadela (Brosimum gaudichaudii Tréc) exibe frutos do tipo drupa com casca globosa, coloração amarelo-alaranjado e textura verrugosa. O diâmetro varia de quatro a cinco centímetros. A polpa é comestível, carnosa e doce, e envolve até duas sementes de coloração creme. Possui quantidades consideráveis de compostos bioativos, como o ácido ascórbico e compostos fenólicos, além de elevados teores de carotenoides, principalmente licopeno e $\beta$-caroteno, apresentando características antioxidantes (Conceição et al., 2012; Alves, 2013; Jesus, 2014; Abreu, 2015).

\subsection{Baru}

O baru (Dipteryx alata Vog.) é uma leguminosa do tipo drupa, com formato ovalado e suavemente achatado, com coloração marrom, tegumento externo liso e brilhante, e possui apenas uma amêndoa comestível (Guimarães et al., 2012; Canuto, 2015; Almeida et al., 2016). A frutificação ocorre entre os meses de setembro e outubro. O fruto possui altos teores de ácidos graxos, principalmente de ácido oleico ( $\omega-9)$ e ácido linoleico ( $\omega-6)$, acompanhados pelo ácido palmítico (Louredo et al., 2014). A amêndoa possui comprimento que pode variar de 1,0 a 2,6 cm, largura de 0,9 a $1,3 \mathrm{~cm}$ e espessura de 0,7 a $1,0 \mathrm{~cm}$, sendo rica em lipídeos (40\%), com grande quantidade de gorduras insaturadas e de importância para o consumo humano, pois reduz os níveis de LDL do colesterol; apresenta 30\% de sua composição em forma de proteínas, além de conter diversos minerais, como ferro, zinco, potássio e cálcio (Louredo et al., 2014; Oliveira et al., 2017). O óleo proveniente da semente de baru apresenta teores de $\alpha$-tocoferol e $\gamma$-tocoferol referentes a 5\% e 4,3\% respectivamente, os quais possuem ação antioxidante (Maciel Junior, 2010).

\subsection{Bocaiuva}

Os frutos da bocaiuva (Acrocomia aculeata) são do tipo drupa, apresentando coloração que varia de amarelo ao alaranjado, formato globoso de diâmetro de 2,5 a 5,0 cm. A frutificação da bocaiuva ocorre durante o ano inteiro, sendo que o período de amadurecimento dos frutos ocorre principalmente entre os meses de setembro e janeiro (Vieira et al., 2012). Possui mesocarpo mucilaginoso e fibroso, paladar suavemente adocicado e endocarpo duro e denso, com cerca de $3 \mathrm{~mm}$ de espessura, envolvendo a polpa oleosa (Sanjinez-Argandoña \& Chuba, 2011). Esta polpa contém aproximadamente $49 \%$ de umidade e teores significativos de carboidratos digeríveis (31\%), fibras alimentares (13,76\%), lipídeos (17\%), proteínas (2\%), minerais $(1,7 \%)$ e valor energético $\left(167,67 \mathrm{kcal} .100 \mathrm{~g}^{-1}\right)$. A amêndoa proporciona alta quantidade de lipídios $(51,7 \%)$, proteínas $(17,6 \%)$ e fibras alimentares $(15,8 \%)$, sendo rica em minerais, como potássio, fosforo e cálcio (Ferreira et al., 2013).

\section{Aproveitamento dos frutos do cerrado}

Diante das características e propriedades encontradas nos frutos do Cerrado descritos acima, este tópico irá abordar alguns estudos mostrando a utilização e a potencialidade dos frutos desse bioma na indústria de alimentos.

\subsection{Pequi}

O pequi possui sabor único, podendo ser utilizado em produtos como licores, doces, conservas de frutas, cozido com arroz, adicionado ao hambúrguer, além da extração de óleo, obtido a partir da sua polpa ou amêndoa, para aplicação na agroindústria ou indústria de alimentos (Sousa et al., 2014).

Em estudo realizado por Cardoso et al. (2014), os autores testaram a adição de 5,5\% de farinha de pequi em barra de cereais, a fim de aproveitar um resíduo gerado, agregar valor ao produto e produzir um alimento nutritivo, funcional e saboroso, uma vez que a casca do pequi apresenta altas concentrações de fibras. Os 
autores concluíram que a barra de cereal se apresentou mais nutritiva e saborosa, além de possuir propriedades funcionais em função do aumento no teor de fibra alimentar $(8 \mathrm{~g} / 100 \mathrm{~g})$.

Segundo Siqueira et al. (2012), a aplicação da pectina de farinha de casca de pequi, obtida com concentração de ácido cítrico de $2 \%$, a 94 minutos e $84^{\circ} \mathrm{C}$, é viável em geleias light.

Devido ao grande teor de lipídeos encontrado no pequi, o uso combinado com outras fontes de óleos tornase promissor para a produção de biodiesel, como forma de redução de poluentes e uso de fonte renovável de biocombustíveis (Cremonez et al., 2016). Machado et al. (2015) obtiveram um extrato aquoso de pequi através da concentração dos carotenoides e compostos fenólicos por ultrafiltração e nanofiltração por membranas, resultando em um produto final com alto teor de componentes bioativos para aplicações funcionais e com elevada capacidade antioxidante.

\subsection{Buriti}

Com altos teores de compostos antioxidantes, o buriti é fonte de carotenoides, vitamina $\mathrm{C}$ e compostos fenólicos, podendo ser aplicado em diferentes produtos na indústria de alimentos, atribuindo valor nutricional, viabilizando também a inovação de produtos com aspectos saudáveis (Rodrigues et al., 2016). Visto isso, o buriti pode ser aplicado na produção de produtos tais como polpa, doces, geleias, sorvetes, néctares, corantes e antioxidantes, e na elaboração de farinha a partir da desidratação da polpa (Garcia et al., 2017; Santos et al., 2011).

Aquino et al. (2012a) aplicaram diferentes quantidades do óleo de buriti em biscoitos do tipo Cookies, objetivando aumentar o consumo de vitamina A na merenda escolar, agregando valor nutricional ao produto. Garcia et al. (2015) elaboraram um néctar de buriti, com características funcionais, uma vez que apresenta baixo valor energético e fonte de vitaminas, como opção de um produto com alto teor nutricional no mercado.

\subsection{Mangaba}

A mangaba possui aroma agradável e grande quantidade de nutrientes, porém muito perecível no seu estádio de maturação avançado, dificultando o consumo in natura. Desta forma, torna-se promissor o processamento do fruto para a elaboração de sorvetes, sucos, licores e vinagres, aumentando sua vida útil (Oliveira Júnior et al., 2016).

Segundo estudo de Vieira et al. (2017), devido à alta acidez, o fruto pode ser utilizado na produção de geleia. Além disso, o uso da mangaba pode apresentar um bom aproveitamento na produção de sorvetes, pois, além de agregar valor com as características funcionais, tem capacidade de fixação de sabor e inibição da formação de cristais.

Estudo de Souza et al. (2015) verificou a produção de lipase de Aspergillus niger por meio da fermentação em estado sólido de resíduo (farinha de semente de mangaba), com grande importância na biotecnologia aplicada.

\subsection{Cagaita}

A cagaita pode ser aplicada na produção de diferentes produtos que apresentam, como características, Ph ácido, baixa acidez titulável e elevada umidade (Camilo et al., 2014), sendo que a utilização do fruto em bebidas é bastante considerável (Martins et al., 2017). Quando a cagaita é submetida ao processo de fermentação, produz vinagre e álcool (Silva et al., 2015b).

Oliveira (2010) realizou experimento utilizando duas espécies de Saccharomyces cerevisiae, as quais foram empregadas na formulação de uma bebida alcoólica fermentada de cagaita, podendo assim aproveitar e potencializar o uso do fruto. 


\subsection{Araticum}

$\mathrm{O}$ araticum possui grande quantidade de polpa, resultando em alto rendimento, podendo ser consumida in natura ou utilizada no preparo de sucos, licores, doces, geleias, tortas e iogurtes (Silva et al., 2015a).

$\mathrm{O}$ consumo da polpa do araticum é limitado pelo fato de ser um fruto sazonal e com propriedades que a tornam altamente perecível, tais como o $\mathrm{pH}$ (baixa acidez) e que favorecem o crescimento microbiano. Visto essa necessidade, foi elaborado um estudo com alternativas de métodos de conservação eficientes para aplicação na polpa do fruto: branqueamento, pasteurização e congelamento. Foi possível verificar que o armazenamento da polpa em 90 dias foi eficaz, utilizando apenas o binômio branqueamento/congelamento, e para um período superior, o binômio pasteurização/congelamento se torna eficaz, até 180 dias (Silva et al., 2015a).

Arruda et al. (2016) realizaram estudo com o objetivo de produzir doce de leite utilizando polpa de araticum, a fim de desenvolver um novo produto e analisar a aceitação do consumidor, aproveitando as características funcionais da polpa de araticum.

Bernal et al. (2016) realizaram a produção e a análise sensorial de um licor de baixa caloria com adição de polpa de araticum, uma vez que o setor de bebidas do tipo licor tem buscado evoluir tecnologicamente e utilizar sabores diferenciados, podendo agregar valor e também aproveitar diferentes sabores, como é o caso do araticum, além de poder caracterizar a região do Cerrado.

\subsection{Guabiroba}

O fruto da guabiroba possui sabor adocicado e características levemente laxativas, podendo ser consumido tanto in natura quanto em produtos elaborados, como geleias, sorvetes, sucos, doces, pudins e licores (Guimarães, 2016).

No estudo de Cristofel (2014), a elaboração de hambúrguer de tilápia enriquecido com ingrediente funcional e coproduto de guabiroba obteve a coloração alterada, devido à presença de carotenoides no resíduo do fruto. Outro efeito foi o aumento do teor de umidade, devido ao aumento da capacidade de retenção de água pelas fibras, bem como observou-se um incremento no teor deste carboidrato não digerível. Além disso, o coproduto de guabiroba influenciou no retardamento da oxidação lipídica, devido às propriedades antioxidantes dos compostos bioativos presentes no fruto.

\subsection{Murici}

As aplicações relatadas sobre o murici estão concentradas no uso dos extratos em fármacos e cosméticos, sendo que a literatura ainda não apresenta o uso da fruta na comercialização de alimentos. Parte disto se deve à elaboração de produtos de maneira informal e ao próprio consumo in natura.

\subsection{Mama-cadela}

A literatura não aponta o uso da mama-cadela em produtos alimentícios disponíveis comercialmente. No entanto, pelas características intrínsecas do fruto, acredita-se ser viável a aplicação da polpa em produtos de confeitaria, panificação, geleias, doces, sucos, sorvetes e compotas. O fruto também apresenta potencial aplicação na indústria não alimentícia, devido às propriedades antimicrobianas em lesões em pacientes diabéticos (Borges, 2016) e no tratamento do vitiligo (Goulart et al., 2017). 


\subsection{Baru}

O baru pode ser aplicado de diversas formas. As amêndoas podem ser processadas a fim de se obter farinha, a polpa pode ser utilizada na produção de doces e, das sementes, pode-se extrair o óleo; estas podem, também, ser submetidas à torrefação (Oliveira et al., 2014).

Estudo realizado por Ortolan et al. (2016) verificou que o baru pode ser adicionado em bolos e produtos de confeitaria. A adição de $12 \%$ de farinha de baru em bolos do tipo cupcakes resultou em maiores teores de umidade, cinzas, proteínas, lipídios, calorias e fibra alimentar, e redução dos carboidratos digeríveis, quando comparada às formulações convencionais, que possuem cerca de $50 \%$ a mais de farinha de trigo. Freitas et al. (2014) verificaram uma melhoria no aspecto nutricional em biscoitos, devido ao alto teor de fibra alimentar, proteínas, minerais e lipídios.

Segundo Pinho et al. (2015), o enriquecimento de sorvete com amêndoa de baru proporcionou aumento na quantidade de lipídeos, valor calórico total, proteínas e de fibras alimentares, quando comparado ao padrão utilizado no estudo. Quando o sorvete foi submetido à análise sensorial, mais de $85 \%$ dos provadores relataram aceitação quanto a aparência, textura e sabor do sorvete de baru.

Lubas et al. (2016) mostraram que os parâmetros nutricionais de barras de chocolate adicionadas de castanhas de baru foram satisfatórios, com aumento no teor de umidade, resíduo mineral fixo e proteína, e redução no teor de carboidratos totais, utilizando $35 \%$ de amêndoa, tal como menor teor de lipídios e calorias, com adição de $15 \%$ dessa amêndoa.

No estudo de Cruz \& Pertuzatti (2014), foram avaliadas sobremesas lácteas sabor chocolate e baru, em que a utilização de baru apresentou benefícios no valor nutricional do produto, devido ao alto teor de ácido linoleico e à quantidade elevada de minerais provenientes da amêndoa.

\subsection{Bocaiuva}

A bocaiuva é um fruto com potencial para ser aplicado tanto na produção de alimentos quanto na geração de biodiesel (Áscoli et al., 2015). Com o processamento da polpa e da amêndoa de bocaiuva, a fim de extrair óleos, os farelos são obtidos como coprodutos e apresentam proteínas, fibras e carboidratos. Devido às suas propriedades nutricionais e funcionais tecnológicas, à capacidade de absorção de água e gordura, emulsificante, estabilizante e espessante, os frutos podem ser aplicados na indústria. Desta forma, podem ser aproveitados para uso em panificação e massas, na indústria de cárneos, além de suplementação alimentar (Grande \& Cren, 2016).

De acordo com Valério et al. (2014), estudos estão sendo realizados e apontam que a bocaiuva apresenta potencial como fonte de óleos brutos que não necessitam ser refinados para consumo humano, podendo assim unir as propriedades físico-químicas do fruto com processos menos refinados e eficientes, adaptando-se à demanda do mercado, que busca uma alimentação mais saudável.

Estudo de Ferreira et al. (2013) utilizou a bocaiuva em um alimento funcional do tipo shake, rico em minerais, fibras, fonte de vitaminas E e A, realçando as ações antioxidante, anti-inflamatória e quimiopreventiva do fruto.

\section{Considerações finais}

A exploração de frutos do Cerrado apresenta potencial no cenário agroindustrial brasileiro. $\mathrm{O}$ processamento e a aplicação vislumbram agregar valor comercial e nutricional, além de conferir aroma, sabor e cor, características únicas e peculiares destes frutos, que apresentam grande perecibilidade. Além disso, a extração de óleos essenciais e compostos bioativos destas matérias-primas, posteriormente micro ou nanoencapsulados (como tendências e desafios para o melhor aproveitamento e preservação dos componentes), poderá fortalecer o uso dos frutos do Cerrado. Essas aplicações poderão viabilizar a 
comercialização dos frutos, gerando emprego e renda à população local, favorecendo a ampliação industrial, minimizando o desperdício e promovendo a geração de coprodutos.

\section{Referências}

Abreu, P. A. A. (2015). Caracterização dos fatores nutricionais e antinutricionais de sementes de frutos do cerrado (Dissertação de mestrado). Escola de Agronomia, Universidade Federal de Goiás, Goiânia.

Albuquerque, J. S. (2016). Propagação vegetativa de guabiroba (Campomanesia Xanthocarpa Berg.) pelo método de enxertia (Trabalho de conclusão de curso). Curso de Engenharia Florestal, Universidade Federal do Paraná, Curitiba.

Almeida Júnior, E. B., Chaves, L. J., \& Soares, T. N. (2014). Caracterização genética de uma coleção de germoplasma de cagaiteira, uma espécie nativa do cerrado. Bragantia, 73(3), 246-252. http://dx.doi.org/10.1590/1678-4499.0075

Almeida, E. L., Goulart, G. A. S., Claro Neto, S., Chierice, G. O., \& Siqueira, A. B. (2016). Preparação e caracterização de poliuretanos contendo diferentes quantidades de óleo de baru. Polímeros, 26(2), 176-184. http://dx.doi.org/10.1590/01041428.2151

Alves, A. M. (2013). Caracterização física e química, compostos bioativos e capacidade antioxidante de frutas nativas do Cerrado (Dissertação de mestrado). Escola de Agronomia, Universidade Federal de Goiás, Goiânia.

Alves, A. M., Alves, M. S. O., Fernandes, T. O., Naves, R. V., \& Naves, M. M. V. (2013). Caracterização física e química, fenólicos totais e atividade antioxidante da polpa e resíduo de gabiroba. Revista Brasileira de Fruticultura, 35(3), 837-844. http://dx.doi.org/10.1590/S0100-29452013000300021

Angella, F. C. O. (2014). Avaliação da atividade antioxidante em extratos de frutas típicas do Cerrado brasileiro (Dissertação de mestrado). Universidade de São Paulo, São Carlos.

Aquino, J. S., Pessoa, D. C. N. P., Araujo, K. L. G. V., Epaminondas, P. S., Schuler, A. R. P., Souza, A. G., \& Stamford, T. L. (2012a). Refining of buriti oil (Mauritia flexuosa) originated from the Brazilian Cerrado: Physicochemical, thermal-oxidative and nutritional implications. Journal of the Brazilian Chemical Society, 23(2), 212-219.

Aquino, J. S., Pessoa, D. C. N. P., Oliveira, C. E. V., Cavalheiro, J. M. O., \& Stamford, T. L. M. (2012b). Processamento de biscoitos adicionados de óleo de buriti (Mauritia flexuosa $L$.): Uma alternativa para o consumo de alimentos fontes de vitamina A na merenda escolar. Revista de Nutrição, 25(6), 765-774. http://dx.doi.org/10.1590/S1415-52732012000600008

Arruda, H. S., Botrel, D. A., Fernandes, R. V. B., \& Almeida, M. E. F. (2016). Development and sensory evaluation of products containing the Brazilian Savannah fruits araticum (Annona crassiflora Mart.) and cagaita (Eugenia dysenterica Mart.). Brazilian Journal of Food Technology, 19(e2015105), 1-7.

Áscoli, A. A., Pereira, A. C., Costa, E., Jorge, M. H. A., \& Maruyama, W. I. (2015). Ambientes protegidos e substratos para mudas de bocaiuva oriundas do campo. Revista de Agricultura Neotropical, 2(3), 23-28.

http://dx.doi.org/10.32404/rean.v2i3.274

Belisário, C. M., \& Coneglian, R. C. C. (2013). Qualidade de frutos de murici (Byrsonima crassifolia, Malpighiaceae) armazenados sob refrigeração. Global Science and Technology, 6(2), 95-101. http://dx.doi.org/10.14688/1984-3801.v06n02a11

Bernal, O. L. M., Alves, J. G. L. F., Castillo, A. M., Santos, D. K., Guerra, S. H., \& Rivera, H. M. V. (2016). Teste de aceitação sensorial de licores de baixa caloria com polpa de marolo (Anona Crassiflora). In Anais do XXI Congresso Brasileiro de Engenharia Química (ENBEQ). Fortaleza: Universidade Federal do Ceará.

Borges, J. C. (2016). Atividade antimicrobiana de extrato de Brosimum gaudichaudii trécul. contra bactérias isoladas de lesões de pés diabéticos (Dissertação de mestrado). Universidade Federal do Tocantins, Palmas.

Braga, V. P. (2017). Avaliação do encapsulamento de sementes recalcitrantes de Campomanesia adamantium (Cambess) 0. BERG (Dissertação de mestrado). Curso de Agronomia, Universidade Federal de Goiás, Jataí.

Camilo, Y. M. V., Souza, E. R. B., Vera, R., \& Naves, R. V. (2014). Caracterização de frutos e seleção de progênies de cagaiteiras (Eugenia dysenterica DC.). Cientifica, 42(1), 1-10. http://dx.doi.org/10.15361/1984-5529.2014v42n1p1-10

Canuto, D. S. O. (2015). Sementes de Baru (Dipteryx alata Vog.). Revista Conexão Eletrônica, 12(1), 1-12.

Cardoso, A. E. A., Zanelato, E. F. N., Viana, E. S. M., Moreira, A. P. B., \& Cardoso, L. M. (2014). Características físico-químicas da farinha da casca do pequi (Caryocar Brasiliense Camb.) e seu aproveitamento na elaboração de barras de cereais. In Anais do VI Simpósio de Pós-graduação em Análises Clínicas (SIMPAC) (Vol. 6, No. 1, pp. 209-214). São Paulo: Universidade de São Paulo.

Cardoso, L. M. (2011). Araticum, cagaita, jatobá, mangaba e pequi do cerrado de Minas Gerais: Ocorrência e conteúdo de carotenoides e vitaminas (Dissertação de mestrado). Curso de Ciência da Nutrição, Universidade Federal de Viçosa, Viçosa.

Carneiro, T. B., \& Carneiro, J. G. M. (2011). Frutos e polpa desidratada Buriti (Mauritia flexuosa L.): Aspectos físicos, químicos e tecnológicos. Revista Verde, 6(2), 105-111.

Carvalho, A. V., \& Nascimento, W. M. O. (2016). Caracterização físico-química e química da polpa de frutos de muruci. Boletim de Pesquisa e Desenvolvimento, 108, 1-17.

Conceição, G. M. (2012). Caracterização botânica e fitossociologia de uma área de Cerrado, no Maranhão, sob pastejo por bovinos (Tese de doutorado). Universidade Estadual Paulista "Júlio de Mesquita Filho", Jaboticabal.

Conceição, S. J. B., Tomé, P. H. F., Pereira, G. A., Machado, S. A., Morais, D. T., \& Rodrigues, A. P. (2012). Composição química e compostos bioativos de frutos de mama cadela (Brosimum gaudichaudii Tréc.). Horizonte Científico, 6, 379-384. 
Cremonez, P. A., Feroldi, M., Oliveira, C. J., Teleken, J. G., Meier, T. W., Dieter, J., Sampaio, S. C., \& Borsatto, D. (2016). Oxidative stability of biodiesel blends derived from different fatty materials. Industrial Crops and Products, 89, 135-140. http://dx.doi.org/10.1016/j.indcrop.2016.05.004

Cristofel, C. J. (2014). Elaboração de hambúrguer de tilápia (Oreochromis Niloticus) enriquecido com ingrediente funcional e resíduo de guabiroba (Campomanesia Xanthocarpa): Características físicas, químicas e sensoriais (Trabalho de conclusão de curso). Curso de Engenharia de Alimentos, Universidade Federal da Fronteira Sul, Laranjeiras do Sul.

Cruz, M. B., Oliveira, W., Barcia, M. T., \& Konda, P. B. P. (2016). Extração de óleo de polpa de buriti: Perfil de ácidos graxos e capacidade antioxidante. In Anais do XXV Congresso Brasileiro de Ciência e Tecnologia de Alimentos (CBCTA). Gramado: UFRGS.

Cruz, P. N., \& Pertuzatti, P. B. (2014). Sobremesas lácteas sabor chocolate e baru (Dipteryx Alata Vogel): Desenvolvimento e caracterização. In Anais do Congresso Brasileiro de Engenharia Química (pp. 1-8). São Paulo: Blucher.

Damiani, C., Vilas Boas, E. V. B., Asquieri, E. R., Lage, M. E., Oliveira, R. A., Silva, F. A., Pinto, D. M., Rodrigues, L. J., Silva, E. P., \& Paula, N. R. F. (2011). Characterization of fruits from the savanna: Araça (Psidium guinnensis Sw.) and Marolo (Annona crassiflora Mart.). Food Science and Technology, 31(3), 723-729. http://dx.doi.org/10.1590/S010120612011000300026

Ferreira, A. N., Santos, C. P. A., Costa, G. L. A., \& Gebara, K. S. (2013). Utilização do extrato de bocaiúva (Acrocomia aculeata) como um alimento funcional do tipo "shake". Interbio, 7(1), 61-71.

Food and Agriculture Organization of the United Nations - FAO. (2012). World Agriculture towards 2030/2050: the 2012 revision (ESA Working Paper, no. 12-03). Rome: FAO. Recuperado em 10 de setembro de 2017, de http://www.fao.org/3/ap106e/ap106e.pdf

Freitas, C. J., Valente, D. R., \& Cruz, S. P. (2014). Caracterização física, química e sensorial de biscoitos confeccionados com farinha de semente de abóbora (FSA) e farinha de semente de baru (FSB) para celíacos. Demetra: Alimentação, Nutrição \& Saúde, 9(4), 1003-1018.

Garcia, L. G. C., Becker, F. S., \& Damiani, C. (2015). Néctar de buriti (Mauritia flexuosa): A bebida funcional do cerrado. Revista Verde de Agroecologia e Desenvolvimento Sustentável, 10(1), 263-268. http://dx.doi.org/10.18378/rvads.v10i1.2778

Garcia, L. G. C., Guimarães, W. F., Rodovalho, E. C., Peres, N. R. A. A., Becker, F. S., \& Damiani, C. (2017). Geleia de buriti (Mauritia flexuosa): Agregação de valor aos frutos do Cerrado brasileiro. Brazilian Journal of Food Technology, 20(0), 176-186. http://dx.doi.org/10.1590/1981-6723.4316

Gonçalves, K. G., Duarte, G. S. D., \& Tsukamoto Filho, A. A. (2015). Espécies frutíferas do cerrado e seu potencial para os safs. FLOVET: Boletim do Grupo de Pesquisa da Flora, Vegetação e Etnobotânica, 1(7), 64-79. Recuperado em 10 de setembro de 2017, de http://periodicoscientificos.ufmt.br/ojs/index.php/flovet/article/view/3111/2153

Goulart, P. S., Araújo, D. R., Silva, B. O., Vilela, P. B., Alves, A. D., \& Santos, A. L. V. (2017). Mamacadela: A cura do vitiligo está no cerrado. Revista Eletrônica Interdisciplinar, 1(17), 271-278.

Grande, S. C., \& Cren, E. C. (2016). Demanda de proteínas vegetais: Potencialidades e o diferencial dos farelos de macaúba: Revisão. Journal of Chemical Engineering and Chemistry, 2(3), 190-214. http://dx.doi.org/10.18540/2446941602032016190

Guimarães, A. C. G. (2016). Potencial antioxidante de treze frutos de espécies de ocorrência no cerrado por diferentes metodologias (Tese de doutorado). Universidade Federal de Lavras, Lavras.

Guimarães, R. C. A., Favaro, S. P., Viana, A. C. A., Braga Neto, J. A., Neves, V. A., \& Honer, M. R. (2012). Study of the proteins in the defatted flour and protein concentrate of baru nuts (Dipteryx alata Vog). Food Science and Technology, 32(3), 464-470. http://dx.doi.org/10.1590/S0101-20612012005000065

Hansen, O. A. S. (2011). Agregação de valor aos frutos da mangabeira (Hancornia speciosa Gomes): Desenvolvimento e avaliação da estabilidade de néctar e geleia (Dissertação de mestrado). Curso de Ciências Agrárias, Universidade Federal do Recôncavo da Bahia, Cruz das Almas.

Instituto de Tecnologia de Alimentos - ITAL. (2010). Brasil Food Trends 2020 (176 p.). São Paulo: Gráfica Ideal.

Jesus, E. J. (2014). Caracterização de frutos de mama cadela (Brosimumgaudichaudii), ao longo do seu desenvolvimento (Dissertação de mestrado). Instituto Federal de Educação, Ciência e Tecnologia do Triângulo Mineiro, Uberaba.

Leão, D. P., Franca, A. S., Oliveira, L. S., Bastos, R., \& Coimbra, M. A. (2017). Physicochemical characterization, antioxidant capacity, total phenolic and proanthocyanidin content of flours prepared from pequi (Caryocar brasilense Camb.) fruit byproducts. Food Chemistry, 225(15), 146-153. PMid:28193408. http://dx.doi.org/10.1016/j.foodchem.2017.01.027

Lima, A., Silva, A. M. O., Trindade, R. A., Torres, R. P., \& Mancini-Filho, J. (2007). Composição química e compostos bioativos presentes na polpa e na amêndoa do pequi (Caryocar brasiliense, Camb.). Revista Brasileira de Fruticultura, 29(3), 695-698. http://dx.doi.org/10.1590/S0100-29452007000300052

Louredo, E. G., Razia, J. R., Lima, L. P., Silva, V. A., Filgueiras, M. L. M., Oliveira, L. F., \& Oliveira, I. P. (2014). Biscoito tipo cookie enriquecido com baru. Revista Faculdade Montes Belos, 7(1), 16-25.

Lubas, C. C. S., Cândido, C. J., Souza, S. V. S., \& Guimarães, R. C. A. (2016). Qualidade nutricional de barras de chocolate adicionadas de castanhas de baru. Multitemas, 21(49), 181-192. http://dx.doi.org/10.20435/multi.v21i49.812

Luzia, D. M. M. (2012). Propriedades funcionais de óleos extraídos de sementes de frutos do Cerrado brasileiro (Tese de doutorado). Universidade Estadual Paulista, São José do Rio Preto.

Machado, M. T. C., Mello, B. C. B. S., \& Hubinger, M. D. (2015). Evaluation of pequi (Caryocar Brasiliense Camb.) aqueous extract quality processed by membranes. Food and Bioproducts Processing, 95, 304-312.

http://dx.doi.org/10.1016/j.fbp.2014.10.013 
Maciel Junior, S. (2010). Caracterização físico-química, qualidade e estabilidade oxidativa do óleo de Dipteryx alata Vog. (Dissertação de mestrado). Universidade Federal do Rio de Janeiro, Rio de Janeiro.

Maia, J. D. (2016). Avaliação de extratos bioativos de mangaba (Hancornia speciosa) utilizando processos a baixas e altas pressões (Dissertação de mestrado). Curso de Engenharia de Alimentos, Universidade Federal de Santa Catarina, Florianópolis.

Martins, H. D., Perfeito, D. G. A., Silva, A. R., \& Peixoto, N. (2017). Caracterização e estudo da estabilidade física de suco misto adoçado de mangaba e cagaita. Revista de Agricultura Neotropical, 4(2), 81-87. http://dx.doi.org/10.32404/rean.v4i2.1542

Mendes, M. R. A., Munhoz, C. B. R., Silva Júnior, M. C., \& Castro, A. A. J. F. (2012). Vegetation and soil relationship in moist grassland in the National Park of Sete Cidades, Piauí, Brazil. Rodriguésia, 63(4), 971-984. http://dx.doi.org/10.1590/S217578602012000400014

Monteiro, D. C. B., \& Pires, C. R. F. (2016). Avaliação da estabilidade físico-química de geleias de murici armazenadas sob diferentes condições de temperatura e luminosidade. Desafios Revista Interdisciplinar da Universidade Federal do Tocantins, 3(especial), 87-98. http://dx.doi.org/10.20873/uft.2359-3652.2016v3nespp87

Morais, E. C., Patias, S. G. O., Ferreira, N. S. S., Picanço, N. F., Rodrigues, E. C., Nascimento, E., \& Faria, R. A. P. G. (2017). Compostos bioativos e características físico-químicas de polpa de araticum in natura e pasteurizada. Brazilian Journal of Food Technology, 20, e2016142. http://dx.doi.org/10.1590/1981-6723.14216

Morzelle, M. C., Bachiega, P., Souza, E. C., Vilas Boas, E. V. B., \& Lamounier, M. L. (2015). Caracterização química e física de frutos de curriola, gabiroba e murici provenientes do Cerrado brasileiro. Revista Brasileira de Fruticultura, 37(1), 96-103. http://dx.doi.org/10.1590/0100-2945-036/14

Nascimento, R. S. M., Cardoso, J. A., \& Cocozza, F. D. M. (2014). Caracterização física e físico-química de frutos de mangabeira (Hancornia speciosa Gomes) no oeste da Bahia. Revista Brasileira de Engenharia Agrícola e Ambiental, 18(8), 856-860. http://dx.doi.org/10.1590/1807-1929/agriambi.v18n08p856-860

Oliveira Júnior, A. M., Conceição Soares, D. S., Santos, J. T. S., \& Nunes, T. P. (2016). Avaliação de diferentes modelos de secagem para liofilização de mangabas maduras com diferentes diâmetros, através de indicadores de desempenho. Scientia Plena, 12(5), 1-6. http://dx.doi.org/10.14808/sci.plena.2016.054210

Oliveira, D. E. C., Resende, O., \& Costa, L. M. (2017). Efeitos da secagem na coloração dos frutos de baru (Dipteryx alata Voge/). Revista Agro@mbiente On-line, 10(4), 364-370. http://dx.doi.org/10.18227/1982-8470ragro.v10i4.3584

Oliveira, L. C., Costa, E., Cardoso, E. D., Binotti, F., \& Jorge, M. H. A. (2014). Propriedades físicas de sementes de baru em função da secagem. Revista de Agricultura Neotropical, 1(1), 92-96. http://dx.doi.org/10.32404/rean.v1i1.223

Oliveira, M. E. S. (2010). Elaboração de bebida alcoólica fermentada de cagaita (Eugenia dysenterica, DC), empregando leveduras livres e imobilizadas (Dissertação de mestrado). Curso de Microbiologia Agrícola, Universidade Federal de Lavras, Lavras.

Ortolan, A. V., Eing, K. K. C., Santos, M. M. R., Cândido, C. J., \& Novello, D. (2016). Adição de farinha de baru em cupcakes: Caracterização físico-química e sensorial entre crianças. O Mundo da Saúde, 40(2), 213-220. http://dx.doi.org/10.15343/01047809.20164002

Pessôa, P. A. P. (2017). Avaliação das propriedades do óleo de buriti (Mauritia flexuosa L.) e sua aplicação em creme vegetal (Tese de doutorado). Curso de Engenharia e Ciência de Alimentos, Universidade Estadual Paulista "Júlio de Mesquita Filho", São José do Rio Preto.

Pinho, L., Mesquita, D. S. R., Sarmento, A. F., \& Flávio, E. F. (2015). Enriquecimento de sorvete com amêndoa de baru (Dipteryx alata Vogel) e aceitabilidade por consumidores. Revista Unimontes Científica, 17(1), 39-49.

Rodrigues, A. M. C., Bezerra, C. V., Silva, I. Q., \& Silva, M. H. (2016). Propriedades reológicas do suco de buriti (Mauritia flexuosa). Revista Brasileira de Fruticultura, 38(1), 176-186. http://dx.doi.org/10.1590/0100-2945-290/14

Sales, V. F. (2016). Importância da preservação, potencialidades e viabilidade para exploração econômica de frutos de buriti (Monografia). Curso de Agronomia, Universidade de Brasília, Brasília.

Sampaio, M. B. (2011). Boas práticas de manejo para o extrativismo sustentável do buriti. Brasília: Instituto Sociedade, População e Natureza.

Sanjinez-Argandoña, E. J., \& Chuba, C. A. M. (2011). Caracterização biométrica, física e química de frutos da palmeira bocaiuva Acrocomia aculeata (Jacq) Lodd. Revista Brasileira de Fruticultura, 33(3), 1023-1028. http://dx.doi.org/10.1590/S010029452011000300040

Santos, C. A., Ribeiro, R. C., \& Silva, E. V. C. (2011). Elaboração de biscoito de farinha de buriti (Mauritia flexuosa L. f) com e sem adição de aveia (Avena sativa L.). Revista Brasileira de Tecnologia Agroindustrial, 5(1), 262-273. http://dx.doi.org/10.3895/S1981-36862011000100002

Silva, L. L., Cardoso, L. M., \& Pinheiro-Sant'Ana, H. M. (2015a). Influência do branqueamento, pasteurização e congelamento nas características físico-químicas, nos carotenoides e no valor de vitamina A de polpa de araticum (Annona crassiflora Mart.). Revista do Instituto Adolfo Lutz, 74(1), 30-38.

Silva, M. M. M. (2016). Estudo do desenvolvimento fisiológico da cagaita (Eugenia dysenterica) (Dissertação de mestrado). Universidade Federal de Goiás, Goiânia.

Silva, S. M. M., Silva, C. A. G., Bazzo, Y. M. F., Magalhães, P. O., \& Silveira, D. (2015b). Eugenia Dysenterica Mart. Ex DC. (cagaita): Planta brasileira com potencial terapêutico. Infarma Ciências Farmacêuticas, 27(1), 49-95. http://dx.doi.org/10.14450/2318-9312.v27.e1.a2015.pp49-95 
Siqueira, B. S., Alves, L. D., Vasconcelos, P. N., Damiani, C., \& Soares Júnior, M. S. (2012). Pectina extraída de casca de pequi e aplicação em geleia light de manga. Revista Brasileira de Fruticultura, 34(2), 560-567. http://dx.doi.org/10.1590/S010029452012000200030

Soares, L. V., Melo, R., Oliveira, W. S., Souza, P. M., \& Schmiele, M. (2017). Brazilian Cerrado fruits and their potential use in bakery products. In H. Lewis (Ed.), Bread: Consumption, cultural significance and health effects (Chap. 5, pp. 125-160). New York: Nova Publisher.

Sousa, E. P., Queiroz, A. J. M., Figueirêdo, R. M. F., \& Lemos, D. M. (2014). Comportamento reológico e efeito da temperatura da polpa de pequi em diferentes concentrações. Brazilian Journal of Food Technology, 17(3), 226-235. http://dx.doi.org/10.1590/1981-6723.1214

Souza, F. M., Travália, B. M., Lima, A. S., Soares, C. M. F., \& Santana, L. C. L. A. (2015). Produção de lipase de Aspergillus niger e imobilização em membranas de poliétersulfona. In Anais do V Simpósio de Bioquímica e Biotecnologia (SIMBBTEC) (pp. 267-270). São Paulo: Blucher.

Torres, L. R., Santana, F. C., Torres-Leal, F. L., Melo, I. L., Yoshime, L. T., Matos-Neto, E. M., Seelaender, M. C., Araújo, C. M., Cogliati, B., \& Mancini-Filho, J. (2016). Pequi (Caryocar brasiliense Camb.) almond oil attenuates carbon tetrachloride-induced acute hepatic injury in rats: Antioxidant and anti-inflammatory effects. Food Chemistry, 225(2017), 146-153. PMid:27623180.

Valério, P. P., Grande, S. C., Andrade, M. H. C., \& Cren, E. C. (2014). Perspectivas para um novo produto alimentício a base de óleo extraído do fruto da macaúba (Acrocomia aculeata (Jacq. Lodd. ex Mart). In Anais do XX Congresso Brasileiro de Engenharia Química. São Paulo: Blucher. Recuperado em 8 de setembro de 2017, de http://pdf.blucher.com.br.s3-sa-east1.amazonaws.com/chemicalengineeringproceedings /cobeq2014/1229-20369-156821.pdf

Vieira, C. M., Souza, E. R. B., Paula, M. S. P., Naves, R. V., \& Silva, G. D. (2017). Mangabeira (Hancornia speciosa Gomes): Uma frutífera promissora do Brasil. Scientific Electronic Archives, 10, 45-55.

Vieira, S. S., Magriotis, Z. M., Santos, N. A. V., Cardoso, M. G., \& Saczk, A. A. (2012). Macaúba palm (Acrocomia aculeata) cake from biodiesel processing: An efficient and low cost substrate for the adsorption of dyes. Chemical Engineering Journal, 183, 152-161. http://dx.doi.org/10.1016/j.cej.2011.12.047

Weiss, C., \& Santos, M. A. (2012). Logística de distribuição e as perdas ao longo da cadeia produtiva das frutas frescas. In Anais do $9^{\circ}$ Congresso Virtual Brasileiro - Administração (pp. 2). São Paulo: Convima. 